\title{
25 Research Suare \\ Total flavonoids of Chinese herb Rhizoma Drynariae alleviates bone loss in caged laying hens
}

Jie Huang

Huazhong Agricultural University

Zhengwang Yu

Huazhong Agricultural University

Xiaofeng Tong

Huazhong Agricultural University

Lei Zhang

Huazhong Agricultural University

Yanping Hu

Huazhong Agricultural University

Zhongxin Zhou ( $\nabla$ zxzhouwhu@163.com)

Huazhong Agricultural University https://orcid.org/0000-0003-3450-9502

Research article

Keywords: Bone, Laying hens, Low calcium, Osteoporosis, Total flavonoids of Rhizoma Drynariae

Posted Date: December 10th, 2019

DOl: https://doi.org/10.21203/rs.2.18412/v1

License: (c) (i) This work is licensed under a Creative Commons Attribution 4.0 International License.

Read Full License 


\section{Abstract}

Background: Caged layer osteoporosis (CLO) is a common bone metabolic disease and is threatening the modern poultry industry. The objective of this study was to evaluate effects of total flavonoids of Rhizoma Drynariae (TFRD), a Chinese herbal extract, on bone health, egg quality and production performance in caged laying hens. Totals of 144 Lohmann pink-shell laying hens of 32-wk-old were randomly allocated to 4 groups (CON, LCD, TFRD1 and TFRD2, respectively). The CON group was fed a basal diet, the LCD group was fed a low calcium diet (LCD) and the TFRD1 and TFRD2 groups were fed LCD supplemented with 0.5 and $2.0 \mathrm{~g} / \mathrm{kg}$ TFRD, respectively.

Results: Supplying $2.0 \mathrm{~g} / \mathrm{kg}$ TFRD produced protective effects on calcium deficiency-induced bone loss in caged laying hens, including reduced serum levels of alkaline phosphatase and tartrate resistant acid phosphatase activities (both $P<0.05$ ) and osteocalcin content $(P<0.05)$, enhanced femur bone mineral density $(P<0.05)$, improved bone microarchitecture deterioration, down-regulated mRNA expressions of RANKL $(P<0.01)$ and up-regulated RUNX2 and OPG levels (both $P<0.01)$. Furthermore, compared to LCD group, $2.0 \mathrm{~g} / \mathrm{kg}$ TFRD treatment had lower malondialdehyd levels $(P<0.01)$, and higher total antioxidant capacity $(P<0.05)$ and glutathione peroxidase $(P<0.05)$ activities, which contributed to the protective effects of bone loss. TFRD supplementation did not affect egg quality while $2.0 \mathrm{~g} / \mathrm{kg}$ TFRD treatment improved laying rate $(P<0.05)$ and decreased feed conversion ratio $(P<0.05)$.

Conclusions: These findings suggested that TFRD has beneficial effects on bone health and production performance in caged laying hens, which can be used for the prevention of CLO.

\section{Background}

Caged layer osteoporosis (CLO), a common bone metabolic disease, is threatening the modern poultry industry. CLO was first described as "cage layer fatigue" by Couch in 1955[1]. Since then, many strategies, such as improving nutrition, strengthening exercise and drugs treatment, were proposed to prevent CLO [2-4]. However, these strategies have not achieved satisfactory results.

Unlike mammals, birds have a unique skeletal structure, the medullary bone, which acts as a dynamic calcium (Ca) reservoir for eggshell formation [5]. Under the long-term and high-yield laying pressure, the Ca circulation of laying hens is in a negative equilibrium state [6]. This state will absorb a large amount of bone $\mathrm{Ca}$ for the formation of eggshell, which will reduce the quality of structural bones and eventually lead to CLO [7]. Since production performance and eggshell quality are closely related to bone healthy, CLO has caused enormous economic losses and involved in animal welfare issues $[8,9]$.

Medicinal plants have been used to prevent and treat osteoporosis in many countries of the world [10]. Total flavonoids from Rhizoma Drynariae (TFRD), a Chinese herbal product extracted from the dried root of Rhizoma Drynariae, has been developed into a postmarketing Chinese medicine called Qianggu capsule [11]. TFRD has shown good therapeutic effects on osteoporosis in human and animal models [12]. The active monomer composition of TFRD include naringin, naringenin, and neoeriocitrin [13]. 
Previous studies have demonstrated that these composition play a dual role in inhibiting the bone resorption and stimulating bone formation, cause underlying anti-osteoporosis activity by regulating bone metabolism signaling pathways, such as OPG/RANKL/RANK, Wnt/ $\beta$-catenin and BMP pathways [14-16], and finally achieve the goal of prevention and treatment of osteoporosis. However, it is unclear whether TFRD also has ideal preventive effects on CLO. Thus, this study aimed to evaluate effects of TFRD on bone health, egg quality and production performance in caged laying hens.

\section{Results}

\section{Performance of caged laying hens}

As shown in Table 1, low calcium diet (LCD) significantly decreased laying rate $(P<0.01)$ and average egg weight (AEW) $(P<0.05)$, and increased feed conversion ratio (FCR) $(P<0.01)$. Supplying $2.0 \mathrm{~g} / \mathrm{kg}$ TFRD in LCD improved laying rate $(P<0.05)$ and reduced FCR $(P<0.05)$. Average daily feed intake (ADFI) among groups did not differ in the study.

Table 1 Effects of TFRD on production performance of caged laying hens

\section{Egg quality}

Table 2 shows that, the eggshell strength and eggshell thickness of caged laying hens fed LCD gradually decreased during the experiment period. At $9 \mathrm{wk}$, the eggshell strength decreased by $52.6 \%(P<0.01)$ and eggshell thickness decreased by $31.4 \%(P<0.01)$ compared to the CON group. In addition, yolk color also dropped significantly $(P<0.01)$. Compared to the LCD group, the supplementation of TFRD did not affect egg quality.

Table 2 Effects of TFRD on egg quality of caged laying hens

\section{Serum redox of caged laying hens}

The serum redox indicators of the LCD group was significantly affected (Table 3 ), as shown by the malondialdehyde (MDA) $(P<0.01)$ levels was enhanced, and the total antioxidant capacity $(T-A O C)(P<$ $0.01)$, total superoxide dismutase (T-SOD) $(P<0.01)$ and glutathione peroxidase (GSH-Px) $(P<0.01)$ activities were reduced. $0.5 \mathrm{~g} / \mathrm{kg}$ TFRD treatment did not have a significant impact on these indicators. Compared with the LCD group, $2.0 \mathrm{~g} / \mathrm{kg}$ TFRD supplementation had lower MDA levels $(P<0.01)$, and higher T-AOC $(P<0.05)$ and GSH-Px $(P<0.05)$ activities, but not affect T-SOD.

Table 3 Effects of TFRD on serum redox indicators of caged laying hens

\section{Femur and tibia bone mineral density (BMD)}

As shown in Table 4, LCD group had lower BMD of femur $(P<0.01)$ and tibia $(P<0.05)$ compared with the CON group. TFRD supplementation did not affect tibia BMD, while $2.0 \mathrm{~g} / \mathrm{kg}$ TFRD treatment significantly increased the femur BMD $(P<0.05)$. 
Table 4 Effects of TFRD on bone mineral density of caged laying hens

\section{Bone histomorphometry}

Fig. 1 shows that effects of TFRD supplementation on the microstructure of the tibia tissue. In the LCD group, many large absorption cavities was appeared on the cortical bone and the cortical bone area was reduced. The trabecular bone became loose or broken with decreased area. With TFRD treatment, the absorption cavity was reduced and the trabecular bone structure was more complete. In addition, the static parameters of bone histomorphometry were analyzed (Table 5). Compared with the CON group, LCD group had significantly lower cortical area ration $(P<0.05)$, percent trabecular area $(P<0.05)$ and trabecular thickness $(P<0.05)$, and higher trabecular separation $(P<0.05) .2 .0 \mathrm{~g} / \mathrm{kg}$ TFRD treatment enhanced cortical area ration $(P<0.05)$, percent trabecular area $(P<0.05)$ and trabecular thickness $(P<$ $0.05)$, and had a lower trabecular separation $(P<0.05)$ than that of LCD hens. Trabecular number and cortical width among groups did not differ in the study.

Fig. 1 Effects of TFRD on microstructure of tibia tissue of caged laying hens

Table 5 Effects of TFRD on bone histomorphometry parameters of caged laying hens

\section{Serum biomarkers of bone metabolism}

As shown in Table 6, LCD group had higher activities of serum alkaline phosphatase (ALP) $(P<0.01)$ and tartrate resistant acid phosphatase (TRACP) $(P<0.01)$, and osteocalcin $(\mathrm{OCN})$ contents $(P<0.01)$ compared with CON group. $2.0 \mathrm{~g} / \mathrm{kg}$ TFRD treatment significantly reduced ALP and TRACP activities (both $P<0.05)$ and OCN content $(P<0.05)$. Serum Ca levels did not differ in the study.

Table 6 Effects of TFRD on serum bone metabolism biomarkers of caged laying hens

\section{Real-time quantitative PCR of bone related genes}

Fig. 2 shows that, LCD significantly down-regulated mRNA expression of runt related transcription factor 2 (RUNX2) $(P<0.01)$ and osteoprotegerin (OPG) $(P<0.01)$, and up-regulated receptor activator of nuclear factor kappa-B ligand (RANKL) $(P<0.01) .0 .5 \mathrm{~g} / \mathrm{kg}$ TFRD treatment enhanced RUNX2 $(P<0.01)$ and OPG $(P<0.05)$, and decreased RANKL $(P<0.01)$ levels. Compared with the LCD group, TFRD2 had higher RUNX2 $(P<0.01)$ and OPG $(P<0.01)$, and lower RANKL $(P<0.01)$ expression.

Fig. 2 Effects of TFRD on RUNX2, OPG and RANKL mRNA expressions of caged laying hens

\section{Discussion}

In the present study, LCD resulted in a significant reduction in laying rate, average egg weight and eggshell quality in caged laying hens. $\mathrm{Ca}$ is a critical nutrients and laying hens need to mobilize about $2.2 \mathrm{~g}$ of Ca daily for eggshell formation [7]. Long-term Ca deficiency in laying hens will limit the secretion of hormones, leading to reduced or even stopped egg production [17]. Supplying TFRD improved 
production performance reduction of caged laying hens caused by LCD. It has been reported that TFRD has estrogen-like effects [18], which may benefit for the reproductive system of laying hens. However, supplementation with TFRD did not affect the egg quality, which is consistent with the results of previous studies $[19,20]$.

LCD also resulted in elevated serum MDA levels and a significant decrease in antioxidase activity of caged laying hens. Ca is a key second messenger involved in intra- and extracellular signaling pathways [21]. Ca homeostasis in the body is closely related to oxidative stress and dietary Ca modulates oxidative and inflammatory stress in mice and humans [22]. Our results showed that supplementation with TFRD decreased the MDA levels and increased antioxidase activity, which indicated that TFRD can alleviate oxidative stress caused by LCD in caged laying hens. Furthermore, total oxidative/anti-oxidative status are close related to BMD in osteoporosis $[23,24]$. The femur and tibia BMD of caged laying hens fed a LCD was significantly reduced. BMD still is the gold standard for the osteoporosis diagnosis [25]. TFRD treatment increased femur BMD in caged laying hens, which indicated that TFRD have a therapeutic effect on low BMD. Furthermore, bone histomorphology showed that TFRD improved the microstructure of tibia tissue, as evidenced by raised cortical area, percent trabecular area and trabecular thickness. Bone histomorphometry is particularly valuable in analyzing the pathology of different forms of bone diseases and in defining the mechanisms by which drugs affect bone [26]. It provides information that is not available from BMD analysis.

Bone metabolism biomarkers, also known as bone turnover markers (BTMs), are a useful adjunct for the diagnosis and therapeutic monitoring of bone metabolic disorders [27]. BTMs are divided into markers of bone formation or resorption and can provide information that is useful for the management of osteoporosis [28]. ALP was the first marker of bone turnover used for both clinical and research purposes to assess metabolic bone disease [29]. OCN, a vitamin K-dependent protein, is found in the mineralized matrix of bone and could be released into the circulation during bone resorption as well as bone formation [30]. TRACP is a marker of bone resorption which reflects the activity of osteoclasts [31]. Low Ca diets resulted in elevated ALP, OCN, and TRACP, indicating a high rate of bone turnover in caged laying hens. TFRD improved the disorder of BTMs, suggesting that TFRD can affect osteoclast-mediated bone resorption exceeding osteoblast-mediated bone formation, thereby preventing bone loss [32].

Previous studies have demonstrated that TFRD can effectively regulate osteogenesis and osteoclastrelated pathways [12]. RUNX2 is a transcription factor that belongs to the Runx family, and has been shown to be essential for osteoblast differentiation and bone formation [33]. The interaction between RANKL and OPG plays a dominant role in osteoclastogenesis [34]. OPG is secreted by osteoblasts and osteogenic stromal stem cells and protects the skeleton from excessive bone resorption by binding to RANKL and preventing it from interacting with receptor activator of nuclear factor kappa-B (RANK) [35]. TFRD has been proven to inhibite osteoclastogenesis via up-regulating OPG, as well as down-regulating RANKL expression in ovariectomized rats [36]. Our results also indicate that supply of TFRD increased mRNA expression of RUNX2 and OPG and decreased RANKL in caged laying hens. 


\section{Conclusions}

In conclusions, TFRD treatment produced protective effects on Ca deficiency-induced bone loss in caged laying hens, including decreased levels of bone matabolism biomarkers, enhanced femur bone mineral density, improved bone microarchitecture deterioration, and regulated the key gene expressions of bone formation and bone resorption. In addition, supplying TFRD improved production performance reduction and alleviated oxidative stress of caged laying hens caused by low Ca diets. These findings suggested that TFRD has beneficial effects on bone health in caged laying hens, which can be used for the prevention of CLO.

\section{Methods}

\section{Experimental design and diets}

A total of 144 Lohmann pink-shell laying hens of 32-wk-old from a commercial farm in the Hubei province of China were randomly allocated to 4 groups (CON, LCD, TFRD1 and TFRD2) with 6 replications of 6 hens per replication. The CON group was fed a corn-soybean basal diet (Table 7), the LCD group was fed a low calcium diet (LCD) (Table 1) and the TFRD1 and TFRD2 groups were fed LCD supplemented with 0.5 and $2.0 \mathrm{~g} / \mathrm{kg}$ TFRD (Beijing Qihuang Pharmaceutical Manufacturing Co., Ltd), respectively.

Table 7 Basal diet and low calcium diet formulation and nutrient levels

\section{Management and sample collections}

The hens were randomly assigned to cages ( $80 \mathrm{~cm}$-width $\times 80 \mathrm{~cm}$-length $\times 40 \mathrm{~cm}$-height) of 6 hens per cage. The hens were kept in an environmentally controlled room with ad libitum feeding and watering and with the temperature controlled at $22^{\circ} \mathrm{C}$ and $16 \mathrm{~h} / \mathrm{d}$ of illumination throughout the entire experimental period. The experiment lasted $9 \mathrm{wks}$. At the end of the experiment, 6 hens from each group were randomly selected, and blood samples were individually collected from the wing vein and then were centrifuged at $3,000 \mathrm{r} / \mathrm{min}$ for $10 \mathrm{~min}$ at $4{ }^{\circ} \mathrm{C}$ to obtain serum. In addition, hens were euthanized with intravenous sodium pentobarbital at a dose of $100 \mathrm{mg} / \mathrm{kg}$. The criteria for euthanasia were somnolence, akinesia and dyspnea. Then, the femur and tibia were collected from the hens.

\section{Production performance}

The number of eggs and egg weight were recorded daily (at 13:00) throughout experiment on a replication basis and hen-day laying rate was calculated. Average egg weight (AEW) was calculated as the mean weight of all eggs from each replicate. Feed consumption was measured weekly on a replication basis. Average daily feed intake (ADFI) was calculated using the following equation: $A D F I=$ feed consumption ( $\mathrm{g}$ ) / (hen number $\times$ time (d)) and feed conversion ratio (FCR) was calculated as (feed intake / (egg weight $\times$ egg production)).

\section{Egg quality determination}


At 3, 6 and $9 w k, 8$ eggs were randomly selected from each group for egg quality determination. Egg shape index was calculated by diameter/height $\times 100$. The eggs were weighed prior to being cracked. Eggshell strength was evaluated using an egg shell force gauge model II (Robotmation Co., Ltd., Tokyo, Japan). Eggshell thickness was measured on the large end, equatorial region, and small end, respectively, using an eggshell thickness gauge (Robotmation Co., Ltd., Tokyo, Japan). Egg weight, albumen height, yolk color, and haugh unit were measured by using a digital egg tester (DET-6000, Nabel Co., Ltd, Kyoto, Japan).

\section{Serum redox assessment}

The activities of total antioxidant capacity (T-AOC) contents, total superoxide dismutase (T-SOD), glutathione peroxidase (GSH-Px), and the contents of malondialdehyde (MDA) in serum were analyzed using analysis kits (Nanjing Jiancheng Bioengineering Institute, Nanjing, China) according to the manufacturer's instructions.

\section{Bone mineral density (BMD) measurement}

The BMD of femur and tibia was measured with dual energy X-ray absorptiometry (InAlyzer, MEDIKORS Inc., Korea). The detection sensitivity of the instrument was $0.001 \mathrm{~g} / \mathrm{cm}^{2}$. A standard calibration block was used to calibrate the device before measurements were made, according to the operator's manual.

\section{Bone histomorphology}

The left tibias were removed from hens and fixed overnight at $4^{\circ} \mathrm{C}$ in $4 \%$ paraformaldehyde, then decalcified and embedded into paraffin. Serial sections $(5 \mu \mathrm{m})$ were cut and stained with Goldner Trichrome stain. In addition, the static parameters of bone histomorphometry were analyzed.

\section{Bone metabolism biomarkers analysis}

The activities of alkaline phosphatase (ALP), tartrate resistant acid phosphatase (TRACP) and the contents of $\mathrm{Ca}$ in serum were measured by specific assay kits from the Nanjing Jiancheng Bioengineering Institute of China. The concentrations of osteocalcin $(\mathrm{OCN})$ in serum were measured with the use of ELISA kits (CH50029; Bio-Swamp, China) according to the manufacturer's instructions.

\section{Total RNA extraction and real-time quantitative PCR of bone related genes}

According to the manufacturer's protocol, the total RNA was extracted from the tissues of the femur by adding TRIzol Reagent (Invitrogen, USA). After extraction with chloroform, isopropanol was added to make the RNA precipitate. After washing with 75\% ethanol, the RNA was eluted in ribonuclease-free water. The cDNA was synthesized using ABScript II RT Master Mix (ABclonal Technology, Wuhan, China). The forward and reverse primer sequences for runt related transcription factor 2 (RUNX2), osteoprotegerin (OPG) and receptor activator of nuclear factor kappa-B ligand (RANKL) were designed based on available sequences on the NCBI GenBank and are listed in Table 8. $\beta$-actin was chosen as an internal standard to 
control for normalization purposes. Quantitation of the mRNA level by QPCR was performed on a realtime PCR system using iTaq Universal SYBR Green Supermix (Bio-Rad, Richmond, CA, USA). The threshold cycle $\left(\mathrm{C}_{\mathrm{T}}\right)$ indicated the fractional cycle number at which the amount of amplified target reached a fixed threshold, so we can obtain the relative gene expression level by the $2^{-\Delta \Delta C}$ method for fold induction. All PCR operations were performed in triplicate.

Table 8 Primers used for the quantitative polymerase chain reaction

\section{Statistical Analysis}

Data were analyzed using the SPSS statistical software (SPSS version 22.0, for windows, SPSS Inc., Chicago, IL). The t-test and one-way ANOVA were used for the analysis of group differences. For measures that presented heterogeneous variances, a Welch-ANOVA was employed. Post hoc analyses were performed by means of Bonferroni test or Games-Howell post hoc test (following the WelchANOVA) to characterize the significant effects. Differences were considered statistically significant at $P<$ 0.05 . The results are presented as the means \pm SD.

\section{Declarations}

\section{Acknowledgements}

The authors are grateful to the staff of Hubei Chenke Agriculture and Animal Husbandry Group Co., Ltd. for their assistance in conducting the experiments.

\section{Funding}

This work was supported by the National Key Research and Development Program of China (No. 2017YFD0502200, No. 2016YFD0501210), Fundamental Research Funds for the Central Universities (No. 2662014BQ023). The funding body provided monetary support only, and the design of the study including collection, analysis, and interpretation of data, and in writing the manuscript were not influenced by the funders.

\section{Authors' contributions}

ZXZ and JH: designed the experiments; JH and LZ: performed the experiments; XFT, ZWY and YPH: assisted with sampling and laboratory analyses; JH and ZXZ: wrote the manuscript; ZXZ: had primary responsibility for the final content; and all authors: read and approved the final manuscript.

\section{Availability of data and materials}

The datasets used and/or analyzed during the current study are available from the corresponding author on reasonable request. 
All experimental procedures involving the use of animals was approved by the Institutional Animal Care and Use Committee of Huazhong Agricultural University, Wuhan, China.

\section{Consent for publication}

Not applicable.

\section{Competing interests}

The authors declare that they have no competing interests.

\section{Abbreviations}

TFRD: Total flavonoids of Rhizoma Drynariae; Ca: Calcium; CLO: Caged layer osteoporosis; LCD: Low calcium diet; AEW: Average egg weight; ADFI: Average daily feed intake; FCR: Feed conversion ratio; MDA: Malondialdehyde; T-AOC: Total antioxidant capacity; T-SOD: Total superoxide dismutase; GSH-Px: Glutathione peroxidase; BMD: Bone mineral density; ALP: Alkaline phosphatase; TRACP: Tartrate resistant acid phosphatase; OCN: Osteocalcin; RUNX2: runt related transcription factor 2; OPG: osteoprotegerin; RANKL: receptor activator of nuclear factor kappa-B ligand; BTMs: Bone turnover markers

\section{References}

1. Couch JR. Cage layer fatigue. Feed Age. 1955;5:55-7.

2. Aguado E, Pascaretti-Grizon F, Goyenvalle E, Audran M, Chappard D. Bone mass and bone quality are altered by hypoactivity in the chicken. Plos One. 2015;10:e116763.

3. Casey-Trott TM, Korver DR, Guerin MT, Sandilands V, Torrey S, Widowski TM. Opportunities for exercise during pullet rearing, part ii: long-term effects on bone characteristics of adult laying hens at the end-of-lay. Poult Sci. 2017;96:2518-27.

4. Olgun 0 , Aygun A. Nutritional factors affecting the breaking strength of bone in laying hens. World Poultry Sci J. 2016;72:821-32.

5. Whitehead CC. Overview of bone biology in the egg-laying hen. Poult Sci. 2004;83:193-9.

6. Kim WK, Bloomfield SA, Sugiyama T, Ricke SC. Concepts and methods for understanding bone metabolism in laying hens. World Poultry Sci J. 2012;68:71-82.

7. Rodriguez-Navarro AB, McCormack HM, Fleming RH, Alvarez-Lloret P, Romero-Pastor J, DominguezGasca N, et al. Influence of physical activity on tibial bone material properties in laying hens. J Struct Biol. 2018;201:36-45.

8. Riber AB, Casey-Trott TM, Herskin MS. The influence of keel bone damage on welfare of laying hens. Front Vet Sci. 2018;5:6.

9. Toscano M, Booth F, Richards G, Brown S, Karcher D, Tarlton J. Modeling collisions in laying hens as a tool to identify causative factors for keel bone fractures and means to reduce their occurrence and 
severity. Plos One. 2018;13:e200025.

10. Li C, Li Q, Liu R, Niu Y, Pan Y, Zhai Y, et al. Medicinal herbs in the prevention and treatment of osteoporosis. Am J Chinese Med. 2014;42:1-22.

11. Wei X, Xu A, Shen H, Xie Y. Qianggu capsule for the treatment of primary osteoporosis: evidence from a chinese patent medicine. Bmc Complem Altern M. 2017;17:108.

12. Zhang $Y$, Jiang J, Shen $H$, Chai $Y$, Wei X, Xie Y. Total flavonoids from rhizoma drynariae (gusuibu) for treating osteoporotic fractures: implication in clinical practice. Drug Des Dev Ther. 2017;11:1881-90.

13. Liu L, Qu W, Liang J. Progress on chemical constituents and biological activities of drynaria fortunei. Strait Pharmaceutical Journal. 2012;24:4-7.

14. Yao W, Zhang $H$, Jiang $X$, Mehmood K, Iqbal M, Li A, et al. Effect of total flavonoids of rhizoma drynariae on tibial dyschondroplasia by regulating bmp-2 and runx2 expression in chickens. Front Pharmacol. 2018;9:1251.

15. Wang D, Ma W, Wang F, Dong J, Wang D, Sun B, et al. Stimulation of wnt/ $\beta$-catenin signaling to improve bone development by naringin via interacting with ampk and akt. Cell Physiol Biochem. 2015;36:1563-76.

16. Wang W, Wu C, Tian B, Liu X, Zhai Z, Qu X, et al. The inhibition of rankl-induced osteoclastogenesis through the suppression of p38 signaling pathway by naringenin and attenuation of titaniumparticle-induced osteolysis. Int J Mol Sci. 2014;15:21913-34.

17. Lin X, Liu Y, Xie C, Wu X, Yin Y. Circadian rhythms and dynamic dietary calcium feeding affect laying performance, calcium and phosphorus levels in laying hens. Biol Rhythm Res. 2018;49:227-36.

18. Wong K, Pang W, Wang X, Mok S, Lai W, Chow H, et al. Drynaria fortunei-derived total flavonoid fraction and isolated compounds exert oestrogen-like protective effects in bone. Brit $\mathrm{J}$ Nutr. 2013;110:475-85.

19. Goliomytis M, Simitzis P, Papalexi A, Veneti N, Hager-Theodorides AL, Charismiadou MA, et al. Influence of citrus flavonoids on laying hen performance, inflammatory immune response, egg quality and yolk oxidative stability. Brit Poultry Sci. 2019;60:1-7.

20. Iskender H, Yenice G, Dokumacioglu E, Kaynar O, Hayirli A, Kaya A. Comparison of the effects of dietary supplementation of flavonoids on laying hen performance, egg quality and egg nutrient profile. Brit Poultry Sci. 2017;58:550-6.

21. Sharaf MS, Van den Heuvel MR, Stevens D, Kamunde C. Zinc and calcium modulate mitochondrial redox state and morphofunctional integrity. Free Radical Bio Med. 2015;84:142-53.

22. Zemel MB, Sun X. Dietary calcium and dairy products modulate oxidative and inflammatory stress in mice and humans. J Nutr. 2008;138:1047-52.

23. Altindag O, Erel O, Soran N, Celik H, Selek S. Total oxidative/anti-oxidative status and relation to bone mineral density in osteoporosis. Rheumatol Int. 2008;28:317-21.

24. Wilson CB. Oxidative stress and osteoporosis. Nat Rev Endocrinol. 2014;10:3. 
25. Lorentzon M, Cummings SR. Osteoporosis: the evolution of a diagnosis. J Intern Med. 2015;277:65061.

26. Compston J, Skingle L, Dempster DW. Bone histomorphometry. In: Vitamin D: Biochemistry, Physiology and Diagnostics. Academic Press; 2018. p. 959-73.

27. Greenblatt MB, Tsai JN, Wein MN. Bone turnover markers in the diagnosis and monitoring of metabolic bone disease. Clin Chem. 2017;63:464-74.

28. Morris HA, Eastell R, Jorgensen NR, Cavalier E, Vasikaran S, Chubb S, et al. Clinical usefulness of bone turnover marker concentrations in osteoporosis. Clin Chim Acta. 2017;467:34-41.

29. Chapurlat RD, Confavreux CB. Novel biological markers of bone: from bone metabolism to bone physiology. Rheumatology. 2016;55:1714-25.

30. Naylor K, Eastell R. Bone turnover markers: use in osteoporosis. Nat Rev Rheumatol. 2012;8: 379-89.

31. Linder CH, Ek-Rylander B, Krumpel M, Norgård M, Narisawa S, Millán JL, et al. Bone alkaline phosphatase and tartrate-resistant acid phosphatase: potential co-regulators of bone mineralization. Calcified Tissue Int. 2017;101:92-101.

32. Song S, Zhai Y, Li C, Yu Q, Lu Y, Zhang Y, et al. Effects of total flavonoids from drynariae rhizoma prevent bone loss in vivo and in vitro. Bone reports. 2016;5:262-73.

33. Vimalraj S, Arumugam B, Miranda PJ, Selvamurugan N. Runx2: structure, function, and phosphorylation in osteoblast differentiation. Int J Biol Macromol. 2015;78:202-8.

34. Tanaka $H$, Mine T, Ogasa $H$, Taguchi T, Liang CT. Expression of rankl/opg during bone remodeling in vivo. Biochem Bioph Res Co. 2011;411:690-4.

35. Martin TJ, Sims NA. Rankl/opg; Critical role in bone physiology. Rev Endocr Metab Dis. 2015;16:1319.

36. Yu F, Lv Q, Tong Z, Song W, Zhao Z, Yuan B, et al. Effect of total flavonoids from drynaria rhizome on bone loss in ovariectomized rats. Trop J Pharm Res. 2019;18:1285-9.

\section{Tables}

Table 1 Effects of TFRD on production performance of caged laying hens. Data are presented as means $(\mathrm{SD})^{\mathrm{a}}$

\begin{tabular}{lcccc}
\hline Items & $\mathrm{CON}^{\mathrm{b}}$ & $\mathrm{LCD}$ & TFRD1 & TFRD2 \\
\hline Laying rate, \% & $93.68 \pm 2.01$ & $66.79 \pm 3.13^{* *}$ & $71.55 \pm 3.11$ & $79.98 \pm 5.27^{\#}$ \\
AEW, g & $61.51 \pm 0.96$ & $57.81 \pm 1.22^{*}$ & $59.26 \pm 0.50$ & $57.35 \pm 0.63$ \\
\hline ADFI, g & $119.52 \pm 0.30$ & $119.22 \pm 0.19$ & $119.12 \pm 0.48$ & $119.32 \pm 0.28$ \\
\hline FCR & $2.08 \pm 0.01$ & $3.10 \pm 0.20^{* *}$ & $2.81 \pm 0.14$ & $2.64 \pm 0.16^{\#}$ \\
\hline
\end{tabular}

${ }^{\mathrm{a}}$ Means were calculated on $\mathrm{n}=6$ replicates (6 hens per replicate) per treatment. 
$\mathrm{b}_{\mathrm{TFRD}}$, total flavonoids of Rhizoma Drynariae; CON, basal diet; LCD, low calcium diet; TFRD1, low calcium diet supplemented with $0.5 \mathrm{~g} / \mathrm{kg}$ TFRD; TFRD2, low calcium diet supplemented with $2.0 \mathrm{~g} / \mathrm{kg}$ TFRD; AEW, average egg weight; ADFI, average daily feed intake; FCR, feed conversion ratio.

${ }^{*} P<0.05,{ }^{* *} P<0.01$ vs. CON; ${ }^{\#} P<0.05,{ }^{\# \#} P<0.01$ vs. LCD.

Table 2 Effects of TFRD on egg quality of caged laying hens. Data are presented as means $(\mathrm{SD})^{\mathrm{a}}$

\begin{tabular}{|c|c|c|c|}
\hline Items & $\mathrm{CON}^{\mathrm{b}}$ & LCD & TFRD1 \\
\hline \multicolumn{4}{|c|}{ Egg shape index } \\
\hline $3 \mathrm{wk}$ & $1.29 \pm 0.03$ & $1.30 \pm 0.04$ & $1.31 \pm 0.031 .31 \pm 0.06$ \\
\hline $6 \mathrm{wk}$ & $1.29 \pm 0.05$ & $1.29 \pm 0.04$ & $1.29 \pm 0.041 .33 \pm 0.04$ \\
\hline $9 \mathrm{wk}$ & $1.30 \pm 0.02$ & $1.29 \pm 0.04$ & $1.30 \pm 0.021 .33 \pm 0.04$ \\
\hline \multicolumn{4}{|c|}{ Eggshell strength, N } \\
\hline $3 \mathrm{wk}$ & $45.15 \pm 8.22$ & $32.67 \pm 8.64^{*}$ & $32.83 \pm 5.3032 .23 \pm 5.52$ \\
\hline $6 \mathrm{wk}$ & $43.21 \pm 7.57$ & $25.74 \pm 8.98^{* *}$ & $23.76 \pm 5.4724 .19 \pm 6.69$ \\
\hline $9 \mathrm{wk}$ & $49.90 \pm 8.27$ & $23.63 \pm 3.93^{* *}$ & $21.14 \pm 2.1924 .06 \pm 3.93$ \\
\hline \multicolumn{4}{|c|}{ Eggshell thickness, mm } \\
\hline $3 \mathrm{wk}$ & $0.34 \pm 0.02$ & $0.31 \pm 0.02^{*}$ & $0.30 \pm 0.04 \quad 0.29 \pm 0.02$ \\
\hline $6 \mathrm{wk}$ & $0.36 \pm 0.03$ & $0.27 \pm 0.05^{* *}$ & $0.25 \pm 0.02 \quad 0.26 \pm 0.04$ \\
\hline $9 \mathrm{wk}$ & $0.35 \pm 0.02$ & $0.24 \pm 0.03^{* *}$ & $0.22 \pm 0.01 \quad 0.24 \pm 0.02$ \\
\hline \multicolumn{4}{|l|}{ Yolk color } \\
\hline $3 \mathrm{wk}$ & $14.59 \pm 0.57$ & $7.51 \pm 0.77^{* *}$ & $7.64 \pm 0.667 .59 \pm 0.54$ \\
\hline $6 \mathrm{wk}$ & $14.58 \pm 0.43$ & $7.85 \pm 0.53^{* *}$ & $7.80 \pm 0.84 \quad 8.19 \pm 0.79$ \\
\hline $9 \mathrm{wk}$ & $13.96 \pm 0.36$ & $7.98 \pm 0.41^{* *}$ & $7.98 \pm 0.548 .49 \pm 0.72$ \\
\hline \multicolumn{4}{|c|}{ Albumen height, mm } \\
\hline $3 \mathrm{wk}$ & $8.16 \pm 1.23$ & $7.46 \pm 1.00$ & $8.33 \pm 1.617 .51 \pm 0.78$ \\
\hline $6 \mathrm{wk}$ & $8.29 \pm 1.50$ & $7.80 \pm 1.22$ & $7.69 \pm 1.107 .69 \pm 1.20$ \\
\hline $9 \mathrm{wk}$ & $8.44 \pm 1.78$ & $7.79 \pm 0.97$ & $7.61 \pm 0.878 .09 \pm 1.59$ \\
\hline \multicolumn{4}{|c|}{ Haugh unit } \\
\hline $3 \mathrm{wk}$ & $89.06 \pm 7.36$ & $86.27 \pm 5.15$ & $90.67 \pm 8.8387 .50 \pm 4.29$ \\
\hline $6 \mathrm{wk}$ & $89.86 \pm 7.39$ & $89.31 \pm 6.36$ & $87.78 \pm 6.3488 .72 \pm 6.68$ \\
\hline $9 \mathrm{wk}$ & $90.24 \pm 8.66$ & $88.35 \pm 3.62$ & $86.54 \pm 4.4289 .42 \pm 8.36$ \\
\hline
\end{tabular}

a Means were calculated on $\mathrm{n}=8$ eggs per treatment.

$\mathrm{b}_{\mathrm{TFRD}}$, total flavonoids of Rhizoma Drynariae; CON, basal diet; LCD, low calcium diet; TFRD1, low calcium diet supplemented with $0.5 \mathrm{~g} / \mathrm{kg}$ TFRD; TFRD2, low calcium diet supplemented with $2.0 \mathrm{~g} / \mathrm{kg}$ TFRD. 
${ }^{*} P<0.05,{ }^{* *} P<0.01$ vs. CON; ${ }^{\#} P<0.05,{ }^{\# \#} P<0.01$ vs. LCD.

Table 3 Effects of TFRD on serum antioxidant indicators of caged laying hens. Data are presented as means $(\mathrm{SD})^{\mathrm{a}}$

\begin{tabular}{lcccc}
\hline Items & CON $^{\mathrm{b}}$ & LCD & TFRD1 & TFRD2 \\
\hline MDA, nmol/mL & $3.52 \pm 0.29$ & $5.17 \pm 0.46^{* *}$ & $4.52 \pm 0.39$ & $4.21 \pm 0.56^{\# \#}$ \\
T-AOC, U/mL & $9.44 \pm 1.28$ & $5.62 \pm 0.77^{* *}$ & $6.63 \pm 0.99$ & $7.27 \pm 1.28^{\#}$ \\
T-SOD, U/mL & $267.46 \pm 9.32$ & $218.17 \pm 14.56^{* *}$ & $226.47 \pm 14.82$ & $224.23 \pm 13.23$ \\
GSH-Px, U/mL $949.41 \pm 88.30784 .99 \pm 76.03^{* * 808.74} \pm 108.56918 .67 \pm 75.88^{\#}$ \\
\hline
\end{tabular}

${ }^{\mathrm{a}}$ Means were calculated on $\mathrm{n}=6$ hens per treatment.

$\mathrm{b}_{\mathrm{TFRD}}$, total flavonoids of Rhizoma Drynariae; CON, basal diet; LCD, low calcium diet; TFRD1, low calcium diet supplemented with $0.5 \mathrm{~g} / \mathrm{kg}$ TFRD; TFRD2, low calcium diet supplemented with $2.0 \mathrm{~g} / \mathrm{kg}$ TFRD; MDA, malondialdehyd; T-AOC, total antioxidant capacity; T-SOD, total superoxide dismutase; GSH-Px, glutathione peroxidase.

${ }^{*} P<0.05,{ }^{* *} P<0.01$ vs. CON; ${ }^{\#} P<0.05,{ }^{\# \#} P<0.01$ vs. LCD.

Table 4 Effects of TFRD on bone mineral density of caged laying hens. Data are presented as means $(\mathrm{SD})^{\mathrm{a}}$

\begin{tabular}{|c|c|c|c|c|}
\hline Items & $\mathrm{CON}^{\mathrm{b}}$ & LCD & TFRD1 & TFRD2 \\
\hline \multicolumn{5}{|c|}{ Femur BMD, g/cm $20.36 \pm 0.030 .27 \pm 0.02^{* * 0} 0.28 \pm 0.020 .30 \pm 0.02^{\#}$} \\
\hline Tibia BMD, $\mathrm{g} / \mathrm{cm}^{2}$ & $0.35 \pm 0.0$ & $8 \pm 0$ & & \\
\hline
\end{tabular}

a Means were calculated on $\mathrm{n}=5$ hens per treatment.

$\mathrm{b}_{\mathrm{TFRD}}$, total flavonoids of Rhizoma Drynariae; CON, basal diet; LCD, low calcium diet; TFRD1, low calcium diet supplemented with $0.5 \mathrm{~g} / \mathrm{kg}$ TFRD; TFRD2, low calcium diet supplemented with $2.0 \mathrm{~g} / \mathrm{kg}$ TFRD; BMD, bone mineral density.

${ }^{*} P<0.05,{ }^{* *} P<0.01$ vs. CON; ${ }^{\#} P<0.05,{ }^{\# \#} P<0.01$ vs. LCD.

Table 5 Effects of TFRD on bone histomorphometry parameters of caged laying hens. Data are presented as means (SD) ${ }^{\mathrm{a}}$ 


\begin{tabular}{|c|c|c|c|}
\hline & $U$ & & \\
\hline & \multicolumn{3}{|l|}{$20.20 \pm 2.7813 .21 \pm$} \\
\hline & 02 & $0.39 \pm$ & \\
\hline & \multicolumn{3}{|c|}{ 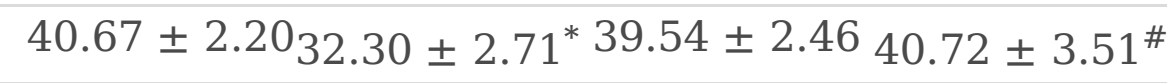 } \\
\hline & \multicolumn{3}{|c|}{$29.08 \pm 2.50_{20.02} \pm 2.57^{*} 27.37 \pm 2.22^{\#} 26.57 \pm 1.61^{\#}$} \\
\hline & \multicolumn{3}{|c|}{$32.57 \pm 1.16_{40.38} \pm 2.86^{*} 35.20 \pm 1.7134 .19 \pm 2.17^{\#}$} \\
\hline & & & \\
\hline
\end{tabular}

${ }^{a}$ Means were calculated on $\mathrm{n}=3$ hens per treatment.

$\mathrm{b}_{\mathrm{TFRD}}$, total flavonoids of Rhizoma Drynariae; CON, basal diet; LCD, low calcium diet; TFRD1, low calcium diet supplemented with $0.5 \mathrm{~g} / \mathrm{kg}$ TFRD; TFRD2, low calcium diet supplemented with 2.0 g/kg TFRD; Ct.Ar, cortical area ration; CW, cortical width; Tb.Ar, percent trabecular area; Tb.Th, trabecular thickness; Tb.Sp, trabecular separation; Tb.N, trabecular number.

${ }^{*} P<0.05,{ }^{* *} P<0.01$ vs. $\mathrm{CON} ;{ }^{\#} P<0.05,{ }^{\# \#} P<0.01$ vs. LCD.

Table 6 Effects of TFRD on serum bone metabolism biomarkers of caged laying hens. Data are presented as means (SD) ${ }^{\mathrm{a}}$

\begin{tabular}{lcccc}
\hline Items & \multicolumn{1}{c}{$\mathrm{CON}^{\mathrm{b}}$} & $\mathrm{LCD}$ & TFRD1 & TFRD2 \\
\hline $\mathrm{ALP}, 100 \mathrm{U} / \mathrm{L}$ & $0.43 \pm 0.10$ & $1.33 \pm 0.19^{* *}$ & $1.26 \pm 0.23$ & $0.98 \pm 0.12^{\#}$ \\
OCN, ng/mL $17.41 \pm 5.2540 .27 \pm 8.26^{* *} 29.88 \pm 5.28$ & $28.81 \pm 6.46^{\#}$ \\
\hline TRACP, U/L & $56.93 \pm 7.1773 .71 \pm 9.38^{* * 63} 63 \pm 7.6459 .45 \pm 8.26^{\#}$ \\
$\mathrm{Ca}, \mathrm{mmol} / \mathrm{L}$ & $2.44 \pm 0.10$ & $2.49 \pm 0.06$ & $2.43 \pm 0.05$ & $2.41 \pm 0.08$ \\
\hline
\end{tabular}

a Means were calculated on $\mathrm{n}=6$ hens per treatment.

$\mathrm{b}_{\mathrm{TFRD}}$, total flavonoids of Rhizoma Drynariae; CON, basal diet; LCD, low calcium diet; TFRD1, low calcium diet supplemented with $0.5 \mathrm{~g} / \mathrm{kg}$ TFRD; TFRD2, low calcium diet supplemented with $2.0 \mathrm{~g} / \mathrm{kg}$ TFRD; ALP, alkaline phosphatase; OCN, osteocalcin; TRACP, tartrate resistant acid phosphatase.

${ }^{*} P<0.05,{ }^{* *} P<0.01$ vs. CON; ${ }^{\#} P<0.05,{ }^{\# \#} P<0.01$ vs. LCD.

Table 7 Basal diet and low calcium diet formulation and nutrient levels 


\begin{tabular}{lll}
\hline Dietary ingredient & \multicolumn{2}{c}{ Content (\%) } \\
& Basal diet & Low calcium diet \\
\hline Corn & 61.86 & 60.97 \\
Soybean meal & 25.84 & 24.05 \\
\hline Bran & 0.50 & 8.00 \\
\hline Limestone & 9.50 & 4.95 \\
\hline Calcium hydrogen phosphate & 1.0 & 0.73 \\
\hline NaCl & 0.30 & 0.30 \\
\hline Premix & 1.0 & 1.00 \\
\hline Total & 100 & 100 \\
\hline Nutrient levels & & \\
\hline Metabolic energy ${ }^{\mathrm{b}}, \mathrm{MJ} / \mathrm{kg}$ & 11.77 & 11.93 \\
\hline Crude protein, \% & 17.25 & 17.58 \\
\hline Calcium, \% & 3.75 & 2.00 \\
\hline Available phosphorus, \% & 0.427 & 0.459 \\
\hline
\end{tabular}

a Premix provided per kilogram of diet: vitamin A, 10,000 IU; vitamin D, 2,000 IU; vitamin $\mathrm{E}, 10 \mathrm{mg}$; vitamin $\mathrm{K}_{3}, 2.0 \mathrm{mg}$; vitamin $\mathrm{B}_{1}, 0.5 \mathrm{mg}$; vitamin $\mathrm{B}_{2}, 4.0 \mathrm{mg}$; vitamin $\mathrm{B}_{6}, 2.0 \mathrm{mg}$; vitamin $\mathrm{B}_{12}, 0.01 \mathrm{mg}$; choline chloride, $400 \mathrm{mg}$; nicotinic acid, $30 \mathrm{mg}$; pantothenic acid, 8.0 mg; folic acid, $0.5 \mathrm{mg}$; iron, 40 mg; copper, $6.0 \mathrm{mg}$; zinc, 50 mg; magnesium, 70 mg; iodine, $0.3 \mathrm{mg}$; and selenium, $0.1 \mathrm{mg}$.

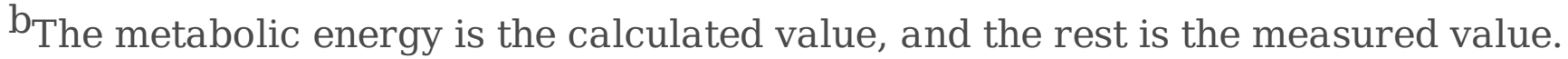

Table 8 Primers used for the quantitative polymerase chain reaction

\begin{tabular}{lccc}
\hline Genes & GenBank ID & Primers sequence (5' to 3') & Products (bp) \\
\hline RUNX2 & FN_204128.1 & F: GATTACAGACCCCAGGCAGG & 75 \\
& R: TGGCTCAAGTAGGACGGGTA \\
OPG & NM_001033641.1F: GTTCCTACTCGTTCCACACC & 115 \\
R: GCTCTTGTGAACTGTGCCTTTG \\
RANKL NM_001083361.1F: AGGAGAAATAAGCCCGAGAA & 108 \\
B-actin NM_205518.1 & F: TTTGTTATGATGCCAGGATGTA \\
\hline & R: CACGATCATGTTTGAGACCTT & 100 \\
\hline
\end{tabular}

a RUNX2, runt related transcription factor 2; OPG, osteoprotegerin; RANKL, receptor activator of nuclear factor kappa-B ligand.

\section{Figures}



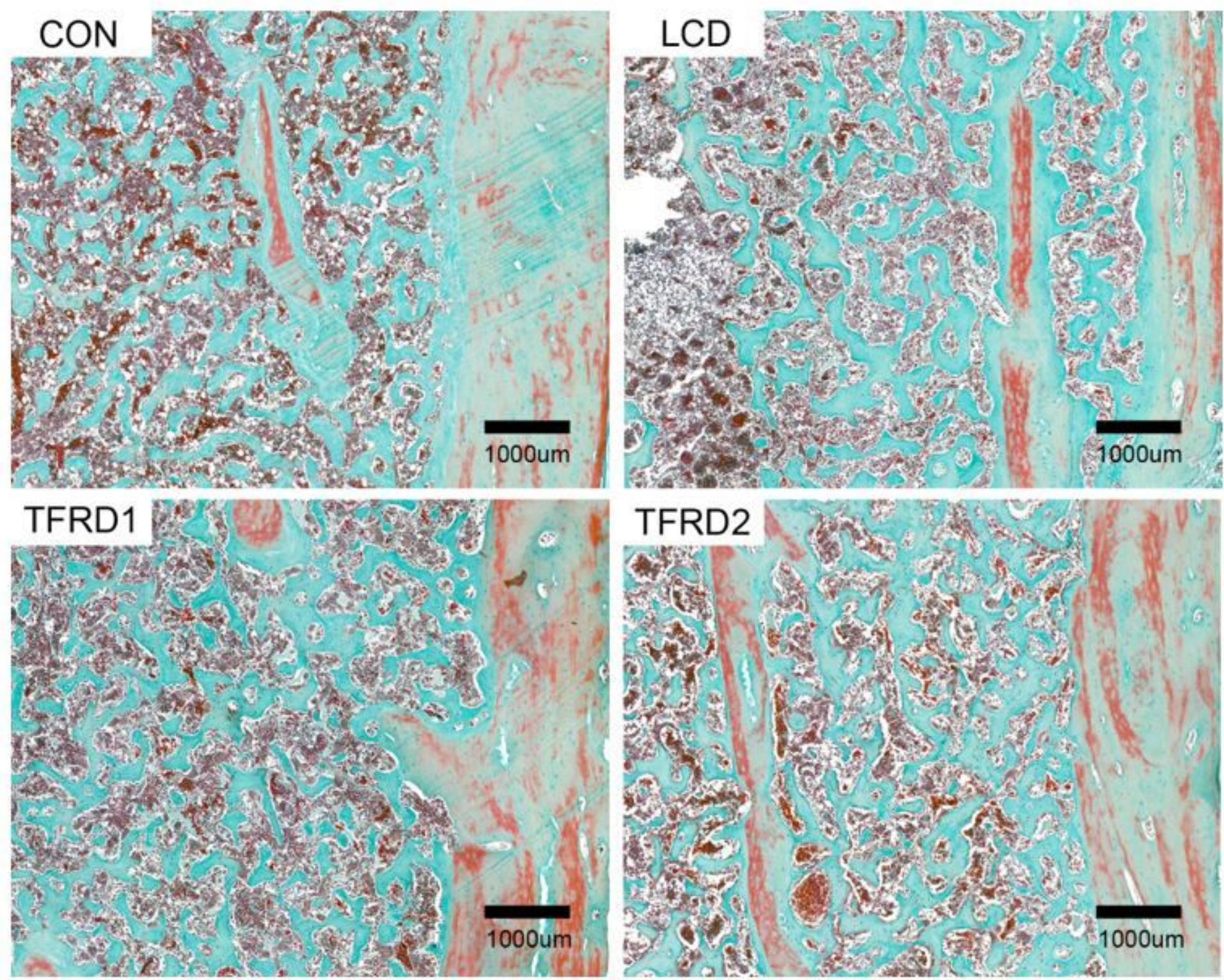

\section{Figure 1}

Effects of TFRD on microstructure of tibia tissue of caged laying hens. Representative images of Goldner's trichrome staining (magnification, 10x). CON, basal diet; LCD, low calcium diet; TFRD, total flavonoids of Rhizoma Drynariae; TFRD1, low calcium diet supplemented with $0.5 \mathrm{~g} / \mathrm{kg}$ TFRD; TFRD2, low calcium diet supplemented with $2.0 \mathrm{~g} / \mathrm{kg}$ TFRD. 


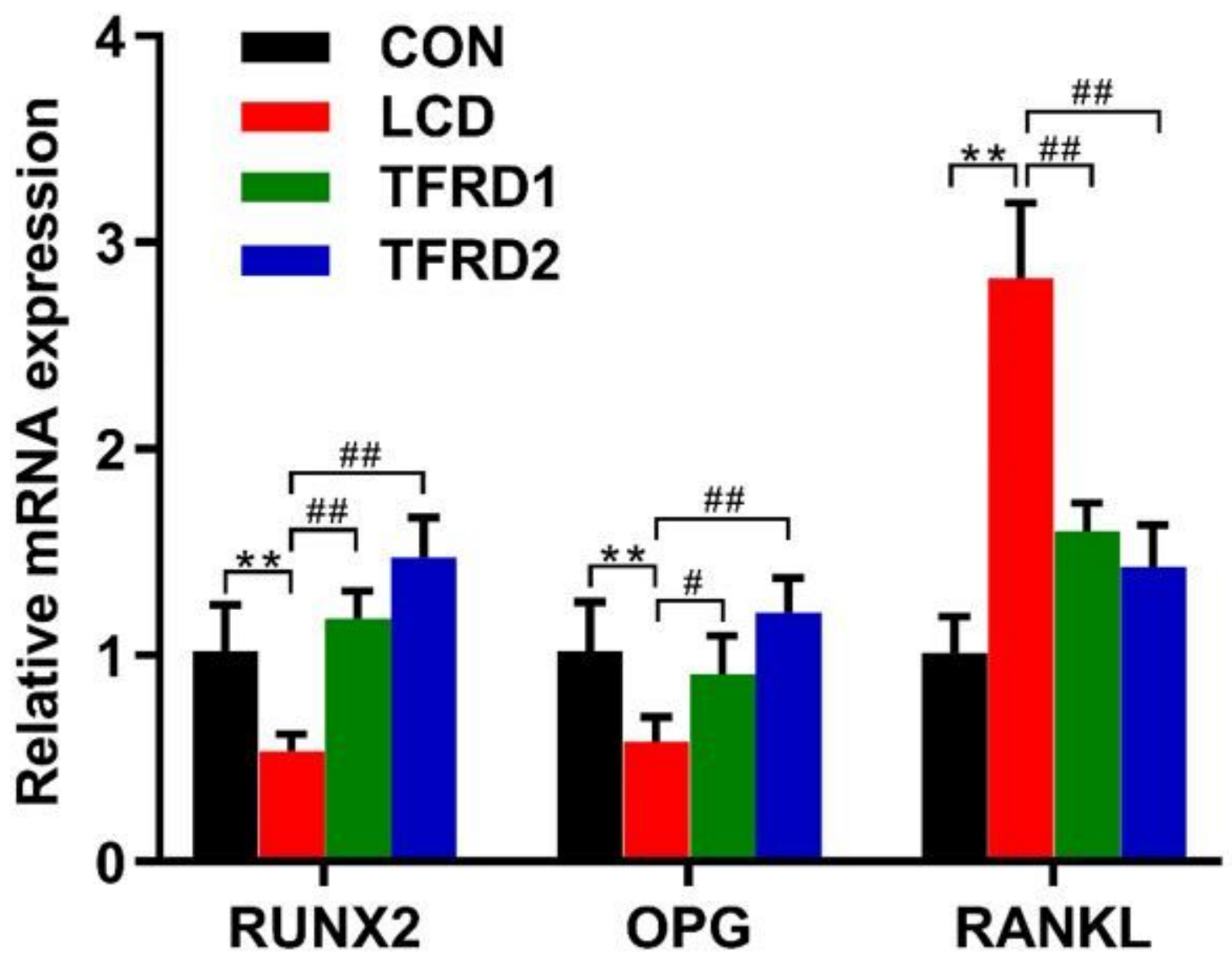

Figure 2

Effects of TFRD on RUNX2, OPG and RANKL mRNA expressions of caged laying hens. Values are means $\pm S D\left(n=5\right.$ hens/treatment). ${ }^{*}<<0.05,{ }^{\star} * P<0.01$ vs. CON; $\# P<0.05$, \#\#P< 0.01 vs. LCD. RUNX2, runt related transcription factor 2; OPG, osteoprotegerin; RANKL, receptor activator of nuclear factor kappa-B ligand; CON, basal diet; LCD, low calcium diet; TFRD1, low calcium diet supplemented with $0.5 \mathrm{~g} / \mathrm{kg}$ TFRD; TFRD2, low calcium diet supplemented with $2.0 \mathrm{~g} / \mathrm{kg}$ TFRD.

\section{Supplementary Files}

This is a list of supplementary files associated with this preprint. Click to download.

- Checklist.pdf 